\title{
Epidemiological characterization of malaria in rural southern Tanzania following China-Tanzania pilot joint malaria control baseline survey
}

\author{
Rashid A. Khatib ${ }^{1 *}$, Prosper P. Chaki ${ }^{1}$, Duo-Quan Wang ${ }^{4}$, Yeromin P. Mlacha ${ }^{1,2,3}$, Michael G. Mihayo',
} Tegemeo Gavana ${ }^{1}$, Ning Xiao ${ }^{4}$, Xiao-Nong Zhou ${ }^{4}$ and Salim Abdullah ${ }^{1}$

\begin{abstract}
Background: Malaria is an important public health problem in Tanzania. The latest national malaria data suggests rebound of the disease in the country. Anopheles arabiensis, a mosquito species renowned for its resilience against existing malaria vector control measures has now outnumbered the endophagic and anthrophilic Anopheles gambiae sensu stricto as the dominant vector. Vector control measures, prophylaxis and case management with artemisininbased combination therapy (ACT) are the main control interventions. This paper presents and discusses the main findings from a baseline household survey that was conducted to determine malaria parasite prevalence and associated risk exposures prior to piloting the T3-initiative of World Health Organization integrated with Chinese malaria control experience aimed at additional reduction of malaria in the area.

Methods: The study was conducted from 4 sub-district divisions in Rufiji District, southern Tanzania: Ikwiriri, Kibiti, Bungu, and Chumbi. Malaria transmission is endemic in the area. It involved 2000 households that were randomly selected from a list of all households that had been registered from the area. Residents in sampled households were interviewed on a range of questions that included use of long-lasting insecticidal nets (LLINs) the night prior to the interview and indicators of socio-economic status. Blood drops were also collected on blood slides that were examined for malaria parasites using microscopes.

Results: The study observed an average malaria parasite prevalence of $13 \%$ across the selected site. Its distribution was 5.6, 12.8, 16.7, and 18\% from Ikwiriri, Kibiti, Bungu, and Chumbi wards, respectively. The corresponding LLIN use discovered were $57.5 \%$ over the district. The highest usage was observed from Ikwiriri at $69.6 \%$ and the lowest from Bungu at $46.3 \%$. A statistically significant variation in parasitaemia between socio-economic quintiles was observed from the study. Males were more parasitaemic than females ( $p$ value $=0.000$ ).

Discussion and conclusion: The findings have been discussed in the light of results from Tanzania Demographic and Health Survey-Malaria Indicator Survey, 2015-2016 and other related studies, together with goals and targets set for malaria control. The paper also discusses the observed parasitaemia in relation to reported LLIN use and its distribution by some important factors as they were explored from the study. It has been concluded that malaria burden is now concentrated on the fringes of the settlements where the poorest section of the population is concentrated and LLIN usage is lower than the national average and targets set by national and global malaria control initiatives.
\end{abstract}

\footnotetext{
*Correspondence: rkhatib@ihi.or.tz

${ }^{1}$ Ifakara Health Institute, Kiko Avenue, Mikocheni, P.O. Box 78373, Dar es

Salaam, United Republic of Tanzania

Full list of author information is available at the end of the article
} 


\section{Background}

Malaria is one of the communicable diseases accounting for major health burden in Tanzania. More than $90 \%$ of the population is at risk of transmission. Tanzania Demographic and Health Survey-Malaria Indicator Survey (TDHS-MIS) 2015-2016 for under-fives using malaria rapid diagnostic test (mRDT) shows average parasite prevalence of $14 \%$ [1]. This figure suggests that that the disease has rebound in the country as prevalence was less than $10 \%$ in 2012 . The main vector species are Anopheles gambiae sensu stricto that has currently been outnumbered by Anopheles arabiensis, and Anopheles funestus is also active in some areas $[2,3]$. The dominant parasite is Plasmodium falciparum [1]. The stated prevalence reported for the country is contrary to milestones set in the country's national strategic plan for malaria that was $5 \%$ in 2016 and a decrease to less than $1 \%$ in 2020 [4]. The major control strategies in place are long-lasting insecticidal nets (LLINs), indoor residual spraying (IRS), larviciding of mosquito breeding sites, intermittent presumptive therapy in pregnant women (IPTp), quality-assured diagnostic testing and treatment of malaria cases with artemisinin-based combination therapy (ACT) [4]. Coverage level for all these interventions fell in 2016 against the progress that had been made in 2012 [1]. Against this background, the China-Tanzania joint malaria control project selected Rufiji district, Coast region in southern Tanzania for piloting the T3-initiative of the World Health Organization-integrated with Chinese malaria control experience aimed at additional reduction of malaria in the area.
The project conducted a baseline household survey across Rufiji district to determine some key metrics of malaria epidemiology in the area. Milestones and targets set by the project will be measured using these baseline parameters. This paper aims at shedding light on these baseline parameters in the area and they will be discussed in the light of the overall malaria condition in the country as highlighted from the TDHS-MIS 2015-2016 [1].

The study aimed at identifying the baseline malaria parasite burden and related risk factors in four wards of Rufiji District, Coast Region.

\section{Methods \\ Study site}

Rufiji is a district located in Coast region, Tanzania. It lies about $200 \mathrm{~km}$ south of Dar es Salaam extending between 7.470 and $8.030^{\circ} \mathrm{S}$ and 38.620 and $39.170^{\circ} \mathrm{E}$ along the Dar es Salaam-Lindi and Mtwara Highway. It occupies a land area of 14,500 sq km (divided into 19 wards and 100 villages), which is almost half of the land of the administrative region to which it belongs and bigger than the two smallest regions on Tanzania mainland. The district is named after the country's largest river, Rufiji River, through which its longest stretch passes, and its huge valley and flood plain defines its ecology, settlement pattern and economic activities.

The district's current population is 248,230 scattered around nearly 100 villages. The major settlements in the district are Utete, the district headquarters and Ikwiriri, Kibiti, Bungu, and Jaribu mpakani, all located along the above-mentioned highway (Fig. 1). The dominant

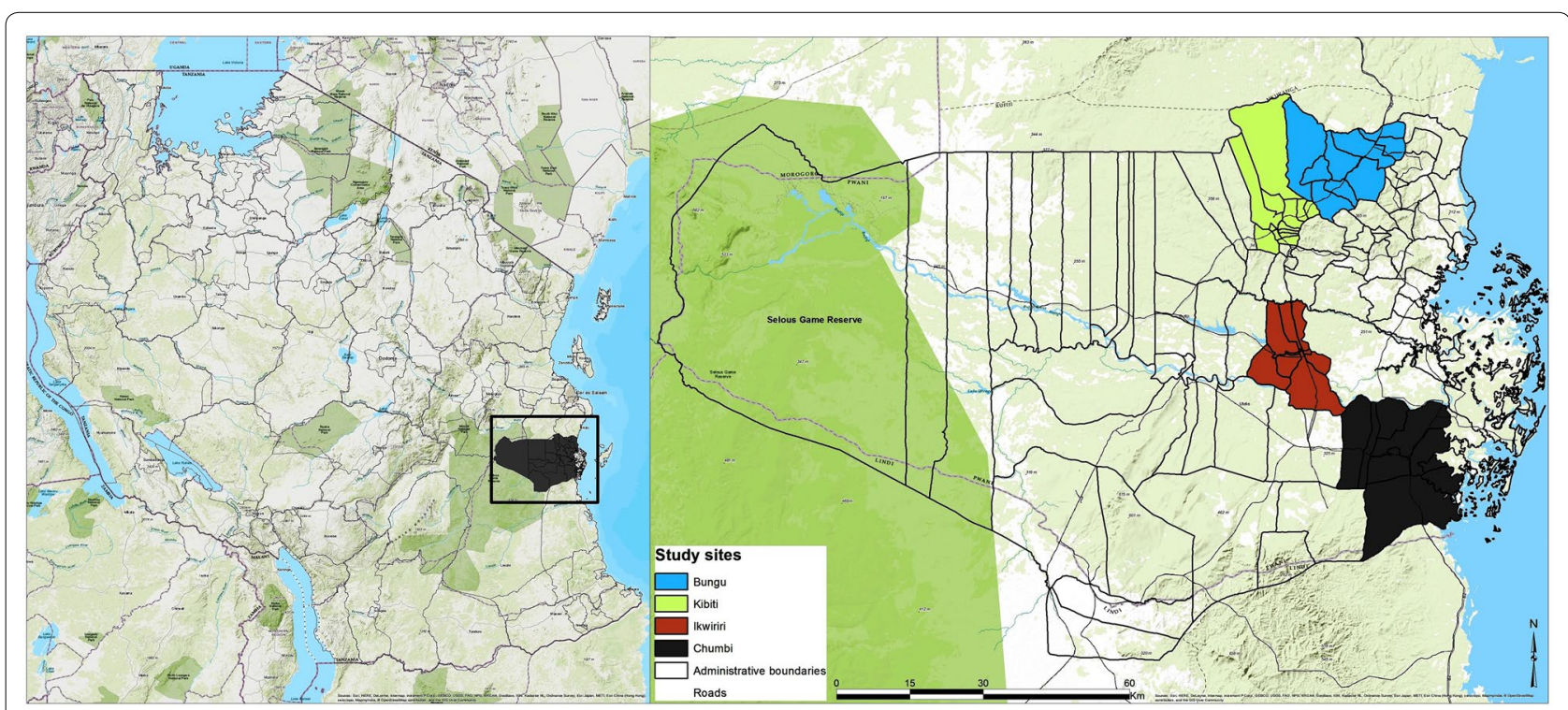

Fig. 1 Study area location 
economic activities in the area are smallholder farming (largely conducted along the river valley), carpentry, artisanal fishing, retailing, and of late, animal keeping. The main crops grown in the area are cassava, rice, maize, fruits, vegetables, cashew nuts, and coconuts.

The district is part of hot, humid, coastal plain with varying tropical climatic conditions. It normally gets rain twice a year; the short season, which is more uncertain, is September and October and the longest rainfall happens from February to May. The river gets most of its water during the long rainfall which normally swells and floods a large area of the valley and lasts up to August and September, which is the period that is most favourable for mosquito breeding and when malaria transmission is most intense.

In terms of epidemiologic and health systems studies, the district is famous for the Demographic and Health Systems Surveillance Systems Site (HDSS). At the time preceding this study, Ifakara Health Institute was hosting one of its two HDSS sites in the district. The other one was Ifakara HDSS site in Kilombero district, Morogoro region. Rufiji HDSS activities were conducted in 6 contagious wards composed of 31 villages to the north of Rufiji River, covering an area of $1813 \mathrm{sq} \mathrm{km}$. The site was staffed by a network of 52 paid field workers and supervisors connected to a team of 118 voluntary informants. It created and maintained a database of all households in the villages and their respective residents who were continuously monitored for vital demographic and health events such as birth, migration and death. Cash flow challenges triggered the suspension of the site activities a short time before the start of this study.

Malaria is the key disease in the district. Its transmission is still endemic. It is most common during and after the period of long rain. It is responsible for the majority of out-patient health facility attendance. Young children and pregnant women are population groups at highest risk of transmission [14]. However, the latest progress in its control has extended the burden to school aged children [10]. Other important ones are waterborne disease, soil-transmitted helminths and lower tract respiratory infections, including TB and AIDs. Health care delivery in the district is dominated by 2 hospitals, 5 health centres and 48 dispensaries. They are predominantly owned by the Government. There are several privately owned and operated retail drug outlets authorized by the Government, known as Accredited Drug Dispensing Outlets (ADDOs) which are commonly concentrated in places with relatively large population centres. They are important for increasing access to some key interventions especially for malaria. Malaria control and delivery strategies depend on same interventions provided by national policy [4].

\section{Study design and procedures}

Data were collected using cross-sectional household survey from September to December 2015 and January 2016. Field activities were conducted in 2 phases.

\section{Phase 1}

The expansion of project activities to areas beyond the HDSS villages forced the team to conduct a census of households and people in the new area before implementation of the survey. The exercise enabled the study to compile a database for sampling selection. The sampling frame was already available in villages covered by HDSS site. The HDSS site wards that were included in the study were Ikwiriri, Mgomba, Umwe, Kibiti, and Bungu. A new non-HDSS ward was added to the study: Chumbi.

\section{Phase II}

Using the database from both the HDSS and the new enumeration, the survey randomly sampled 2000 households from the 6 wards with an estimated 10,000 members. Two modules of questionnaire were prepared. Module 1 was a questionnaire that collected household information, such as household characteristics and asset ownership. This form was addressed to the head of a household and if he was not available, another senior household member was interviewed. The second module targeted every member of the selected household. Once the household was selected, every member of the selected household was interviewed. As for children aged 16 and below, their parents and caretakers consented and were interviewed on their behalf. A total of 9522 individuals, equivalent to $95.5 \%$ of the target population, were interviewed. However, only 7056 individuals, equivalent to $73.5 \%$, were pricked for blood collection. Other people were not pricked because they only accepted to participate in the interviews without consenting to invasive procedures necessary for blood collection. Among the main data collected from the interviews were household asset ownership and household characteristics. They were used to generate household socio-economic status.

Blood drops which were collected were stained on glass slides and stained with Giemsa. They were read by trained microscopists using standard procedures for preparation, interpretation and reporting. Both $P$. falciparum and non-falciparum asexual parasites and gametocytes were identified, but over $98 \%$ of malaria infections in these areas were due to $P$. falciparum and prevalence of non-falciparum infection is not reported. Asexual parasites were quantified by counting number of parasites per 200 white blood cells. Parasite density was estimated by assuming a count of 8000 white blood cells per $\mu \mathrm{l}$ of blood. Five per cent of slides read by each 
Table 1 Observed sample from each selected ward and its distribution by a sex, age group and socio-economic status

\begin{tabular}{|c|c|c|c|c|c|c|c|c|c|c|c|}
\hline \multirow[t]{2}{*}{ Ward } & \multirow[t]{2}{*}{$N=9552$} & \multicolumn{2}{|l|}{ Gender } & \multicolumn{3}{|c|}{ Age groups } & \multicolumn{5}{|c|}{ Socio-economic status } \\
\hline & & $\begin{array}{l}\text { Male n } \\
(\%)\end{array}$ & $\begin{array}{l}\text { Female } \mathrm{n} \\
(\%)\end{array}$ & $<5 r$ & $\begin{array}{l}5-15 \mathrm{n} \\
(\%)\end{array}$ & $>15 \mathrm{n}(\%)$ & $\begin{array}{l}\text { Poorest } \\
\text { n (\%) }\end{array}$ & $\begin{array}{l}\text { Second n } \\
\text { (\%) }\end{array}$ & $\begin{array}{l}\text { Third n } \\
\text { (\%) }\end{array}$ & $\begin{array}{l}\text { Fourth n } \\
\text { (\%) }\end{array}$ & $\begin{array}{l}\text { Least poor } \\
\mathrm{n}(\%)\end{array}$ \\
\hline Ikwiriri & 2595 & $1172(45.2)$ & $1423(54.8)$ & $452(17.4)$ & $792(30.5)$ & $1351(52.1)$ & $300(11.6)$ & $510(19.7)$ & $531(20.5)$ & $508(19.6)$ & $746(28.8)$ \\
\hline Kibiti & 2568 & $1127(44.0)$ & $1441(56.0)$ & $479(18.7)$ & $823(32.1)$ & $1266(49.3)$ & $491(19.1)$ & $394(15.3)$ & $544(21.2)$ & $427(16.6)$ & $712(27.7)$ \\
\hline Bungu & 2303 & $1045(45.4)$ & $1258(54.6)$ & $433(18.8)$ & $779(33.8)$ & $1091(47.4)$ & $347(15.1)$ & $567(24.6)$ & $569(24.7)$ & $557(24.2)$ & $263(11.4)$ \\
\hline Chumbi & 2086 & $1001(48.0)$ & $1085(52.0)$ & $396(19.0)$ & $633(30.4)$ & $1057(50.7)$ & $773(37.1)$ & $445(21.3)$ & $286(13.7)$ & $393(18.8)$ & $189(9.1)$ \\
\hline
\end{tabular}

microscopist were read again by a senior laboratory technician for quality control; discordant readings were consistently less than $14 \%$.

All two-form modules were prepared and pre-tested in Swahili. They were then installed in tablet computers. The data were recorded in these tablets. Members of the field team were recruited from laid-off HDSS staff. They were familiar with the villages and the households that were collected; because they had worked in this kind of study before using tablets computers, it was easier for them to be trained.

\section{Data analysis}

Data sets were transferred into STATA version 10 software (Stata Corp, College Station, TX, USA) for merging, cleaning and performing analyses. To account for unequal probability of selection, all results were weighted (weight $=1$ /probability of selection) and were adjusted for clustering with households as the primary sampling unit. The analysis were done with svy: command in STATA. The main outcome measure was the proportion of observed participants with malaria parasites for the whole study. The prevalence was then compared by sex, age group and socio-economic status (SES). SES was estimated using the scores calculated from the household characteristics and asset ownership that were collected from the study. It was generated using principal component analysis (PCA). The prevalence was also analysed for each ward involved in the study. The corresponding distribution of parasitaemia from each ward and sex, age group and SES were also analysed. Comparison of these outcomes within and between wards and the stated risk factors was made using Chi square test. Multivariable logistic regression was used to assess the importance of the selected risk factors for malaria parasitaemia in the study. The Concentration Index formula by Kakwani et al. [5] as adapted in STATA was used to generate concentration indices and the respective confidence intervals from the study to identify existence of socio-economic inequality in malaria parasite prevalence in the study area. The concentration curves were created using MS Excel version 10.

\section{Results}

Table 1 displays respondents that participated in the study and their distribution by basic socio-economic characteristics. Six wards were involved in the study. However, because of many similarities between Ikwiriri, Umwe and Mgomba wards, as they are all and situated at one contagious location, the analysis pooled them as one ward and designated them as Ikwiriri. Ikwiriri will carry the findings for the 3 wards throughout this paper.

Table 2 displays malaria parasite prevalence observed from the study and its magnitude from each of the 4 wards. It also shows the distribution of the burden by gender, age group and SES. The overall observed prevalence was $13.0 \%$. The highest prevalence was recorded from Chumbi at $18.4 \%$, which was $41 \%$ above the districtwide average. The lowest burden (5.6\%) was observed from Ikwiriri: $43 \%$ below that of the district. According to Table 2, males were in general significantly more parasitaemic than females $(p=0.000)$. The burden was consistently higher for school-aged children, 5-15 years compared to under-fives $(\mathrm{p}=0.000)$. Consistent with many other observations, the findings from this study suggest that parasite prevalence was the lowest among the relatively richest individuals than among the poorest $(\mathrm{p}=0.000)$ (Table 2$)$.

Table 3 presents results generated from multivariate analysis that associates malaria and gender, age group, SES and ITN use. Unlike in univariate analysis as presented in Table 2, where males were more parasitaemic than females $(\mathrm{p}=0.000)$, it is shown in Table 3 that gender had statistically significant relationship with malaria parasitaemia only from Bungu where males were likely to be $40 \%$ more parasitaemic than females (95\% CI 1.1-1.8. It is similarly shown from the table that people above 15 years and above were less likely by $60 \%$ to be infected by malaria parasites compared to under-fives (95\% CI 0.3-0.5). The observation was consistent in all of the wards involved in the study. School-aged children 
(5-15 years) were at higher risk of malaria transmission than under-fives (see Table 3). However, by comparing the two variables according to the wards that the respondents came from, observations were statistically significant only from Kibiti and Bungu.

In terms of SES, the wealthiest individuals in the district were observed to be less vulnerable to malaria parasites compared to the poorest (95\% CI 0.2-0.4). These district-wide results were comparable to those from Kibiti and Bungu where the least poor were less parasitaemic than the poorest by $70 \%$.

The results suggest an overall $60 \%$ reduction in malaria parasitaemia in people sleeping under an LLIN compared to people who do not use them. Protection provided to LLIN users was maintained in every ward selected for the study, except from Chumbi. Fever prevalence also predicted parasitaemia from the study. People that reported fever 14 days preceding interview were twice as parasitaemic as others who did not report the condition.

Table 4 reports LLIN use for the district and from each selected ward and its distribution by gender, age group and SES. Overall, LLIN use from the district was 58\%. Females reported higher LLIN use than males $(p=0.000)$ and children younger than 5 years reported the highest LLIN use of all other age groups $(\mathrm{p}<0.05)$, and schoolaged children had the smallest proportion of LLIN use $(\mathrm{p}=0.000)$. The highest LLIN coverage was observed from Ikwiriri and the lowest was observed from Bungu. Females consistently reported higher LLIN coverage than males in all wards $(\mathrm{p}<0.05)$. School-aged children were least protected in every ward. In terms of wealth quintiles, the least poor segment of the population in every community enjoyed higher ITN coverage than the poorest $(\mathrm{p}<0.05)$.

The concentration curves plotted using data from the national survey as depicted in Fig. 2 lie above the line of perfect equality, suggesting the existence of disproportionate inequality in malaria parasite prevalence against the poorest population in the country. This is supported by the negative CIX $(-0.0098)$ that corroborates the curve. However, since the index is so small it suggests that the concern is not so serious and it can easily be fixed. Figure 3 represents the overall situation from the study and follows the national pattern. In contrast, the CIX is larger away from 0 showing a bigger magnitude of parasitaemia inequality afflicting the poorest population in Rufiji district versus the country. The overall pattern from the study was repeated in all wards (Figs. 4, 5, 6) involved in the study, except Chumbi (Fig. 7). The CIX from all wards showing concentration curves above the line of equality suggests inequality in malaria burden that is more severe than the national average.
Figure 8 rules out the existence of average meaningful socio-economic inequality in LLIN use in the country. The fact that all curves lie below the diagonal supported by positive values in CIX with confidence intervals to the right of 0 from Figs. 9, 10, 11, 12, 13 demonstrates that national data are hiding the true condition of LLIN use inequality in some narrow geographical settings. The figures reveal the weakness of the aggregate data presented in the national survey in evaluating equality of coverage in malaria interventions.

Figures 4, 5, 6, 7 demonstrates concentration curves and indices that depict equal socio-economic inequality based on malaria parasitaemia and ITN coverage from the study. Figure 2 shows the concentration curve lying above the perfect line of equality, which can be interpreted as higher exposure to malaria parasite prevalence for the poorest. The overall magnitude of inequality for the district and across the wards is reflected by concentration indices below $0(-0.1 ; 95 \% \mathrm{CI}-0.21$ to 0.13$)$. This disproportionate distribution of parasitaemia discriminating against the poor was statistically significant in the district. Concentration curves presented in Figs. 2, $3,4,5,6$ lie below the diagonal generated from data observed from the study, and supports other findings from the national survey. The concentration curve from this graph was above $0(0.13 ; 95 \% \mathrm{CI} 0.11-0.15)$.

\section{Discussion}

This is the first study demonstrating malaria parasite prevalence in a narrow geographic setting in Tanzania since the TDHS-MIS 2015-2016 [1]. The average parasitaemia observed from the study was larger than the national average. National malaria prevalence as measured using microscopy reported for 2016 was $6 \%$. However, the national malaria data were limited to under-fives and disaggregated down to only regional level. Pwani, a region to which Rufiji district belongs, reported malaria parasite prevalence for under-fives of only $5.8 \%$. Underfive parasitaemia from this study was $14.8 \%$. This is a large variation between the national and district level data that demonstrates that even if the overall country's malaria burden is in decline as in some countries in Africa [6], the problem is shifting towards certain geographical locations in the same country. This variation was reported even between various regional divisions. Some regions in the central, northeastern and southern highland zones had parasite prevalence lower than $1 \%$ while in regions around Lake Victoria, such as Geita, parasitaemia was $17.7 \%$ [1].

It is here been presented this kind of variation in parasitaemia between the 4 sub-district divisions that were selected for the study. While an average parasitaemia was 


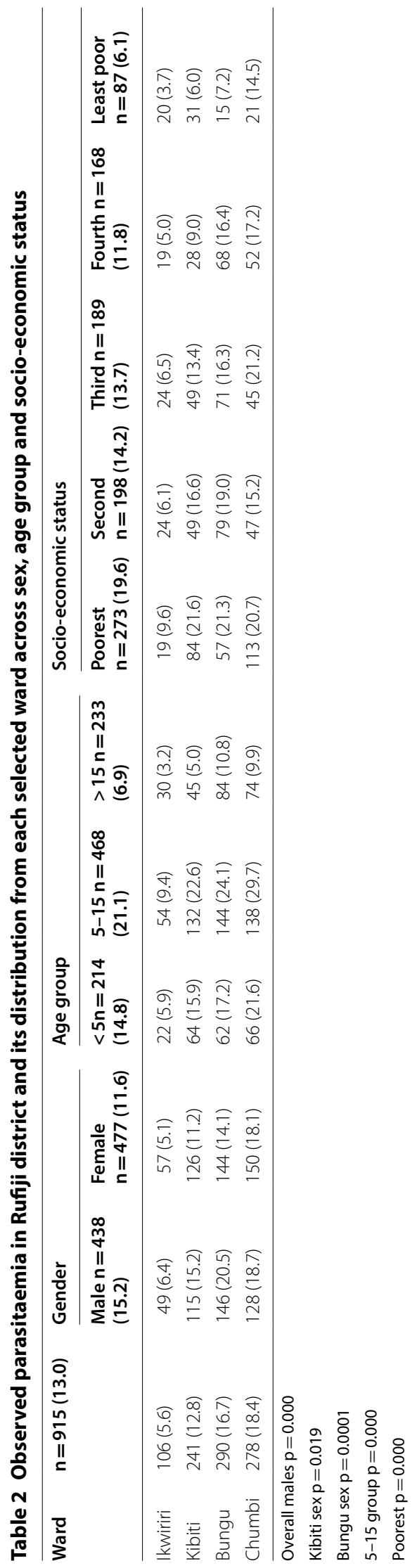


Table 3 Factors related to parasitaemia in Rufiji district by wards

\begin{tabular}{|c|c|c|c|c|c|c|c|c|c|c|c|c|c|c|c|}
\hline & \multicolumn{3}{|c|}{ Districtwide } & \multicolumn{3}{|c|}{ Ikwiriri } & \multicolumn{3}{|c|}{ Kibiti } & \multicolumn{3}{|c|}{ Bungu } & \multicolumn{3}{|c|}{ Chumbi } \\
\hline & OR & $95 \% \mathrm{Cl}$ & p value & OR & $95 \% \mathrm{Cl}$ & p value & OR & $95 \% \mathrm{Cl}$ & $p$ value & OR & $95 \% \mathrm{Cl}$ & p value & OR & $95 \% \mathrm{Cl}$ & $\mathrm{p}$ value \\
\hline \multicolumn{16}{|l|}{ Gender } \\
\hline Female & \multicolumn{15}{|c|}{ Baseline } \\
\hline Male & 1.1 & $1.0-1.3$ & 0.130 & 1.1 & $0.8-1.7$ & 0.500 & 1.1 & $0.8-1.5$ & 0.580 & 1.4 & $1.1-1.8$ & 0.010 & 0.9 & $0.7-1.2$ & 0.572 \\
\hline \multicolumn{16}{|l|}{ Age group } \\
\hline$<5$ & \multicolumn{15}{|c|}{ Baseline } \\
\hline $5-15$ & 1.4 & $1.2-1.8$ & 0.000 & 1.6 & $1.0-2.7$ & 0.056 & 1.5 & $1.03-2.1$ & 0.036 & 1.5 & $1.1-2.2$ & 0.018 & 1.4 & $0.9-2.1$ & 0.096 \\
\hline$>15$ & 0.4 & $0.3-0.5$ & 0.000 & 0.5 & $0.3-1.0$ & 0.045 & 0.3 & $0.1-0.5$ & 0.000 & 0.6 & $0.4-0.9$ & 0.018 & 0.4 & $0.3-0.6$ & 0.000 \\
\hline \multicolumn{16}{|c|}{ Socio-economic status } \\
\hline Poorest & \multicolumn{15}{|c|}{ Baseline } \\
\hline Second & 0.7 & $0.5-0.9$ & 0.007 & 0.6 & $0.3-1.4$ & 0.273 & 0.7 & $0.4-1.2$ & 0.179 & 0.8 & $0.5-1.4$ & 0.474 & 0.7 & $0.4-1.2$ & 0.164 \\
\hline Third & 0.6 & $0.5-0.8$ & 0.005 & 0.7 & $0.3-1.6$ & 0.395 & 0.6 & $0.3-1.0$ & 0.040 & 0.6 & $0.4-1.1$ & 0.097 & 1.1 & $0.7-1.7$ & 0.725 \\
\hline Fourth & 0.5 & $0.4-0.7$ & 0.000 & 0.6 & $0.2-1.3$ & 0.196 & 0.4 & $0.2-0.6$ & 0.001 & 0.7 & $0.4-1.3$ & 0.248 & 0.8 & $0.4-1.6$ & 0.388 \\
\hline Least poor & 0.3 & $0.2-0.4$ & 0.000 & 0.4 & $0.2-1.0$ & 0.043 & 0.3 & $0.2-0.5$ & 0.000 & 0.3 & $0.1-0.7$ & 0.003 & 0.8 & $0.4-1.6$ & 0.535 \\
\hline \multicolumn{16}{|c|}{ Fever presence } \\
\hline No & \multicolumn{15}{|c|}{ Baseline } \\
\hline Yes & 2.4 & $1.9-2.9$ & 0.000 & 2.1 & $1.2-3.7$ & 0.015 & 3.2 & $2.1-4.8$ & 0.000 & 1.8 & $1.2-2.8$ & 0.009 & 1.5 & $1.1-2.1$ & 0.012 \\
\hline \multicolumn{16}{|l|}{ ITN use } \\
\hline No & \multicolumn{15}{|c|}{ Baseline } \\
\hline Yes & 0.6 & $0.5-0.7$ & 0.002 & 0.6 & $0.4-0.9$ & 0.045 & 0.6 & $0.4-0.8$ & 0.001 & 0.6 & $0.4-0.8$ & 0.004 & 0.8 & $0.6-1.1$ & 0.159 \\
\hline
\end{tabular}

13\%, Ikwiriri showed parasite prevalence lower than $6 \%$ and Chumbi, which was the most parasitaemic ward, had a prevalence of $18 \%$. Variation was expanded to include some other factors that were in many other studies associated with malaria transmission. It has been reported that parasitaemia was more common for school-aged children compared to other groups, including under-fives who, together with pregnant women, are still considered to be at highest risk of malaria [6]. It is because of this prevailing knowledge that most malaria-evaluating studies, including malaria indicator surveys, are concentrating on under-fives $[1,7,8]$. The changing malaria risks that are expanding or shifting to older children have been reported in several other studies $[9,10]$. Unequal malaria distribution based on SES has also been highlighted in this study. The disease is most common among poorest groups in the community. Poverty as a risk exposure to malaria has been reported elsewhere [8, 11-14]. The lowest parasitaemia observed from the study was in a ward with largest proportion of least poor, and possessed the largest features of an urban settlement. This observation is consistent with other studies on malaria epidemiology in Africa [15, 16]. It can, therefore, be deduced that malaria is a disease of poverty and is more concentrated on the fringes of a population. Additional progress on control and subsequent elimination requires efforts that will concentrate on the poorer populations in poorer settlements.

Long-lasting insecticidal net use is scaled up as a control tool for vectors responsible for malaria transmission. Many and different programmes have been implemented in Tanzania and in many other places that are at risk of malaria [17-19]. Effective LLIN coverage can provide both personal and community protection against malaria [20-22] even though its validity is counter-indicated by an increasing population of $A n$. arabiensis, a sister species of $A n$. gambiae complex which poses a major setback to existing vector control strategies [23]. It has been shown in a number of studies that LLIN roll out is responsible for the decline of malaria burden observed in many places [24-26]. Three major strategies used for rolling out LLINs in Tanzania are a universal coverage campaign (in 2010-2011), private sector distribution and School Net Programme [1, 17]. From a 2015-2016 survey, an average 50.7\% was collectively achieved for the country [1]. This study has reported higher average LLIN use than national figures. It has been shown that malaria prevalence was not homogenous across the study. With the exception of one location, whose case will be discussed in a separate paragraph, malaria parasite prevalence was inversely related to LLIN use. The burden was largest 


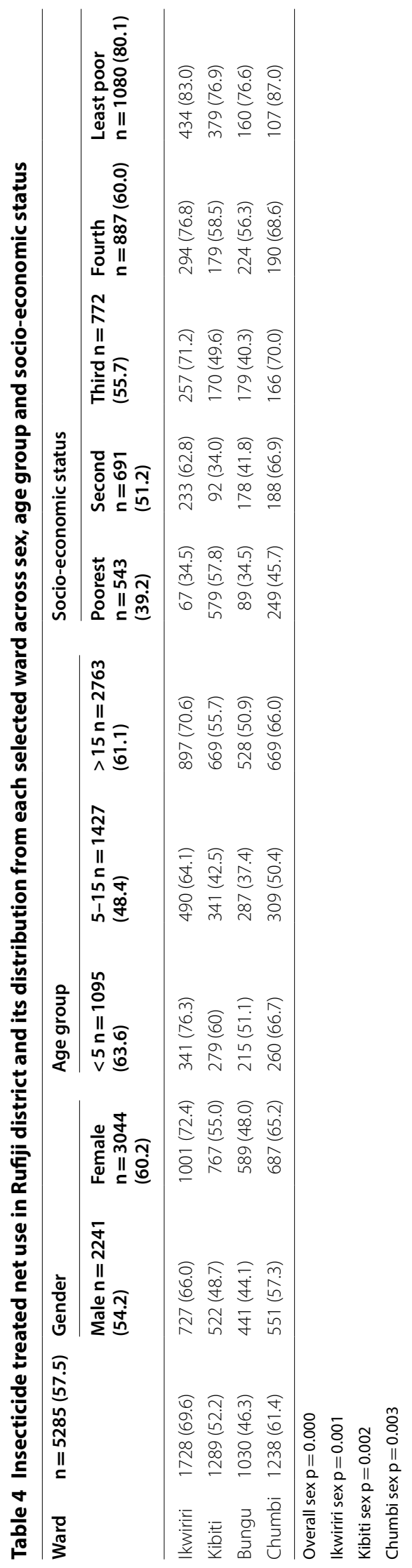




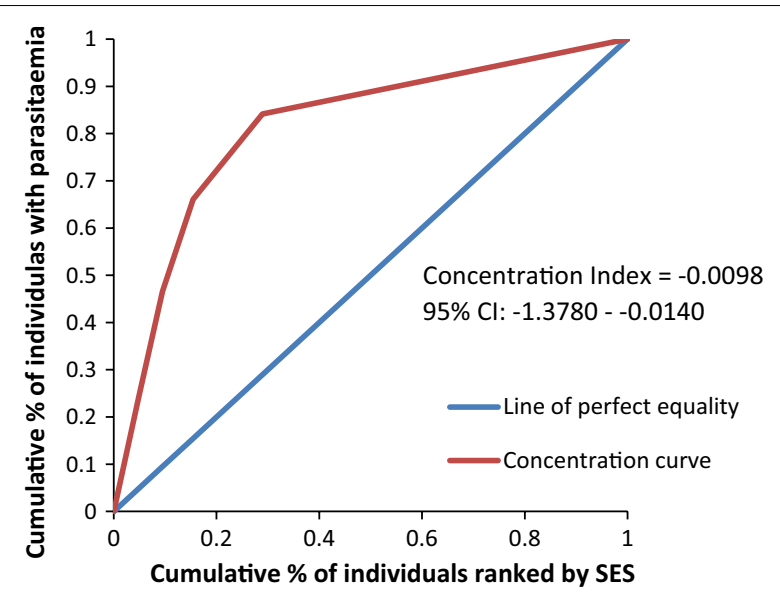

Fig. 2 Socio-economic inequality in malaria parasitaemia as generated from the national malaria survey 2015-2016

where LLIN use was the lowest and it was the lowest where LLIN use was the highest. This observation cannot and is not supported at every place because malaria transmission functions in a multitude of conditions and its control is multifactorial $[8,10]$. A number of studies have demonstrated a significant decline in malaria that was not explained in terms of high LLIN coverage $[8,10]$. Achieving effective coverage, under certain conditions, leads to lower malaria burden. However, there are interventions, including changes in environmental conditions and secular trend, which can bring the malaria burden down independent of LLIN coverage $[27,28]$. For example, a TDHS-MIS report showed that not all regions reported with highest malaria parasite prevalence had the lowest LLIN coverage [1]. Indeed, the Government and its partners strive to achieve LLIN coverage over $80 \%$ by 2020 [29]. This goal has not yet been achieved as the current coverage is $49 \%$ [1].

As for larger parasite burden observed from schoolaged children versus under-fives and other population groups, LLIN follow the same pattern. This age category possessed the lowest LLIN coverage in the study. This observation is supported by results from the TDHSMIS that suggest LLIN use was highest for under-fives and among people living in urban areas [1]. Females were also associated with higher LLIN use than males. The variation can be associated with LLIN distribution programmes targeting under-fives and pregnant women as the existing knowledge singles them out as biologically most vulnerable to malaria transmission [6]. However, it is important for new malaria control plans to be aware of this new reality, as success in sustaining gains and accelerating progress towards end goals in the fight against malaria will depend on clearing parasitaemia from everyone. This is so important for the prevention of onward transmission. Programmes intended to improve malaria interventions for the currently recognized vulnerable groups should be modified to include other groups observed with highest parasitaemia.

It has been stated that a high malaria parasitaemia observed from Chumbi ward presents a paradox for a pattern that shows an association between high LLIN use and low malaria parasitaemia and improved SES and high LLIN coverage. The ward was characterized by the lowest proportion of residents in the highest SES but had higher LLIN use next to Ikwiriri. However, being in the top league of LLIN users was not reflected in parasitaemic status which was the highest of all wards in the study. Various studies have reported that massive roll out of existing malaria vector control interventions have a devastating impact on mosquito species An. gambiae sensu stricto that formerly played a leading role as a malaria vector in Africa [23, 30, 31]. Implementation of LLINs alone or in combination with IRS has successfully reduced the population of this mosquito species bringing many malaria-endemic countries close to goals of malaria control. However, the emergence of An. arabiensis, a sister species of An. gambiae complex, which can survive on both bovine and human blood, poses a new challenge to eliminating malaria. Their susceptibility to control has been attenuated by their ability to feed outdoor whether at dusk or dawn on both humans and animals, in the case of indoor human protection with existing vector control interventions [33]. Being zoophagic, it has been shown that they are more abundant in areas with large animal populations as was the case in Chumbi ward where domestication of animals was one of its major economic activities [23, 32]. A substantial number of residents in the area are pastoralists who tend to spend prolonged hours with their animals which likely exposes them to opportunistic malaria vectors, and which have exhibited insecticide avoidance behaviour. Malaria protection potential of LLINs, whose usage in Chumbi had surpassed several other locations in the study, could hardly be optimized due to conditions discussed. This paradox was not limited to this study area. TDHS-MIS also shows the highest malaria parasite prevalence in regions that are predominantly pastoralists [1]. However, LLIN coverage was higher than in some other regions, reporting relatively low parasitaemia. This could vindicate entomological models demonstrating decreased protection potential of conventional vector control measures in the face of changing dynamics of vector composition $[23,33,34]$.

Many studies have identified inequality as an important barrier to achieving universal coverage of malaria 


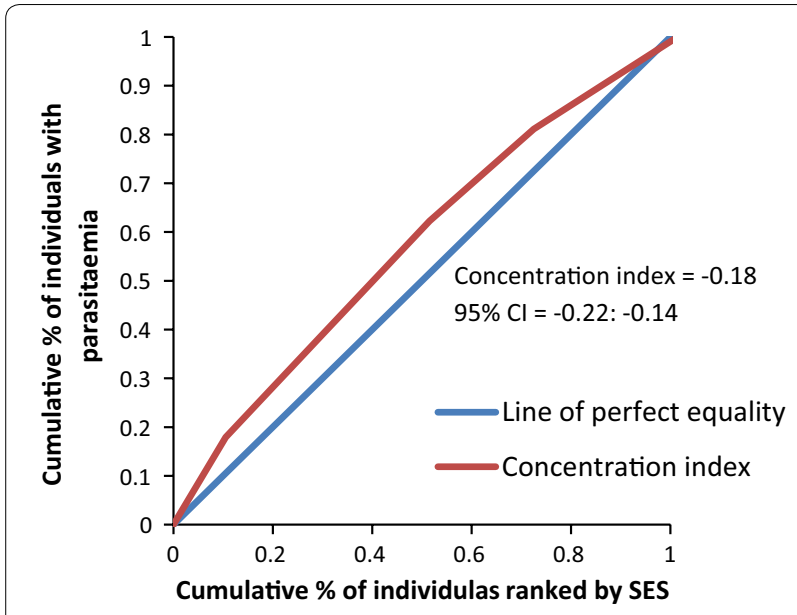

Fig. 3 Socio-economic inequality in malaria parasitaemia in Rufiji generated from the study survey

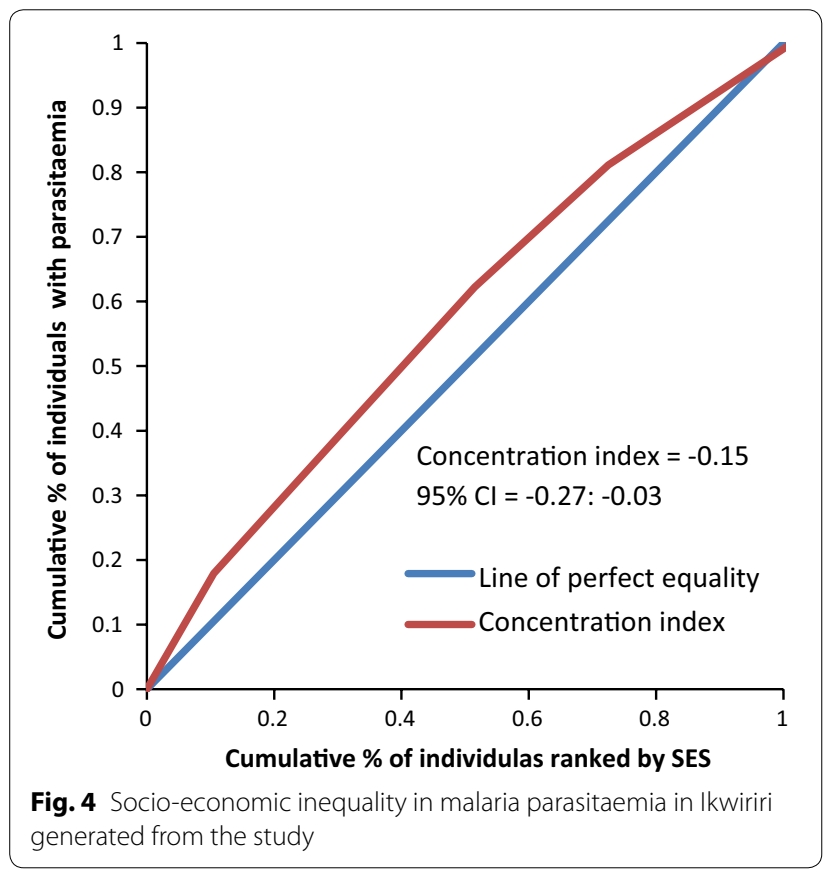

control interventions that is necessary for achieving total control of the disease $[35,36]$. The findings from the study have shown various forms of inequality. The most important one that has consumed enormous resources to fix was inequality based on SES. Logistic regression model presented in results section supported by concentration curves and concentration indices has demonstrated the presence and significant magnitude of wealth-based inequality in parasitaemia and LLIN use. All concentration curves generated using malaria parasitaemia data are hovering above the
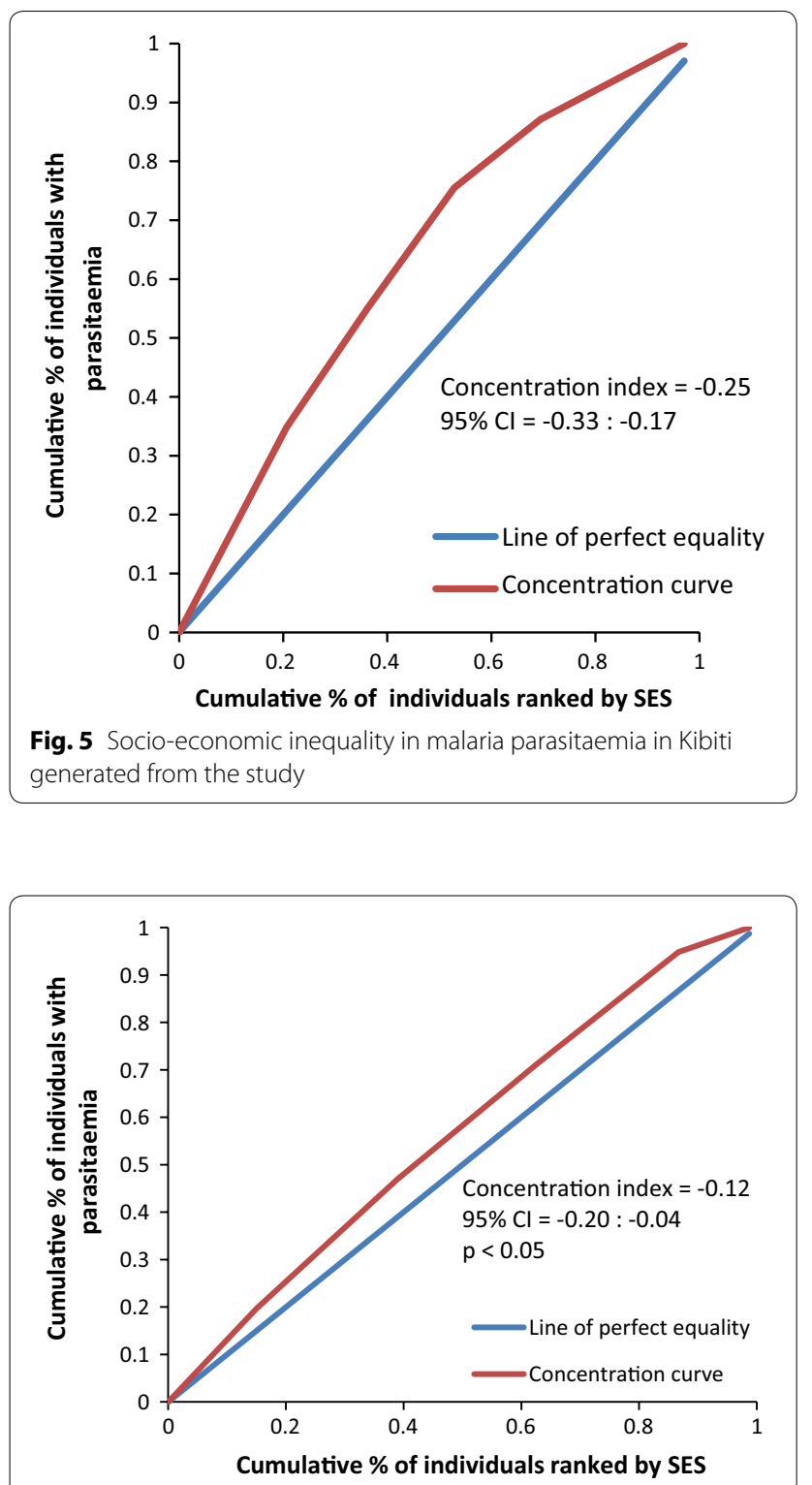

Fig. 6 Socio-economic inequality in malaria parasitaemia in Bungu generated from the study

line of perfect equality. All CIX lie to the left of 0 and can be interpreted that malaria is concentrated in the poorest population. Concentration curve from national malaria data is no different from that observed in this study. Equally important, several evaluation studies conducted in Africa have demonstrated that poverty is a risk factor for malaria [13, 37, 38]. Poor access to malaria control interventions has often been cited as a valid explanation for this. Of all interventions in place, the study paid attention to LLINs only. It showed the lowest LLIN use among the poorest study participants 


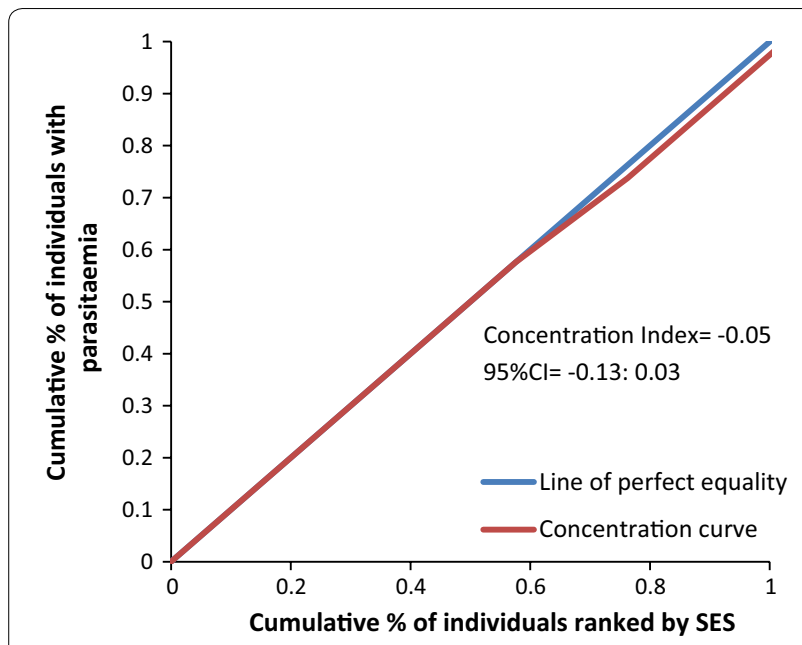

Fig. 7 Socio-economic inequality in malaria parasitaemia in Chumbi generated from the study

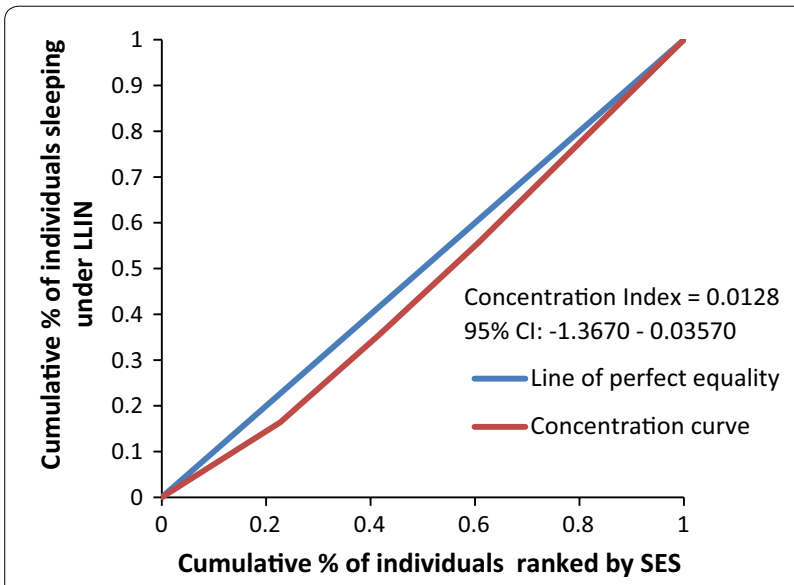

Fig. 8 Socio-economic inequality in LLIN use generated from the national survey 2015-2016

as reflected in concentration curves lying below the diagonal.

The question to ask is, what did happen to the LLIN universal campaign aimed at addressing poverty as an obstacle to access? Is the investment worth pursuing? TDHS-MIS and other studies following up sources of nets providing coverage to different socio-economic groups found that LLINs from mass campaigns were responsible for the largest proportion of nets used by the poorest population [39]. These nets were commonly reported from rural areas. Conversely, private sector distribution was responsible for the majority of nets used by the least poor population [22]. This was commonly reported in urban areas. A source of LLINs in this type of setting is a function of SES that trickles down to urban

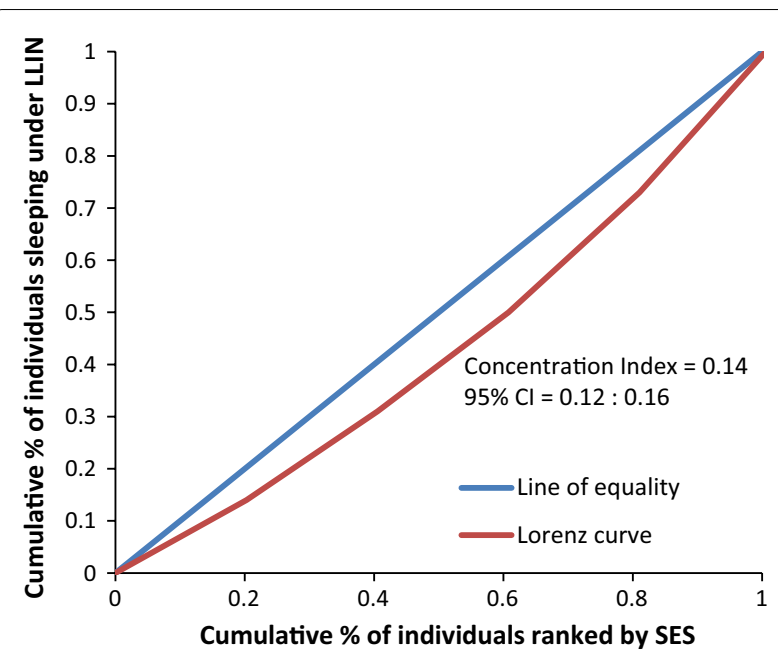

Fig. 9 Socio-economic inequality in LLIN use in Rufiji district generated from the study

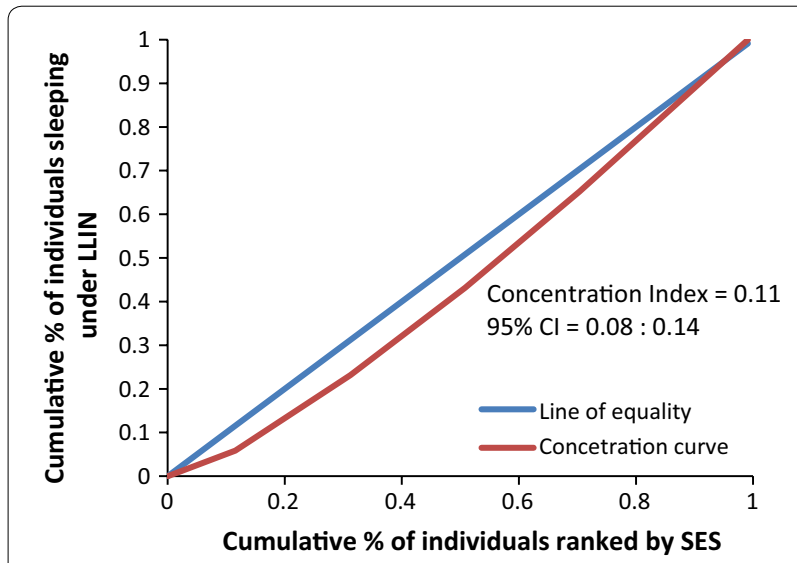

Fig. 10 Socio-economic inequality in LLIN use in Ikwiriri generated from the study

and rural areas. It is evident that mass distribution campaigns are universal and periodic while the private sector is continuous but only active in urban areas. Time intervals between mass campaigns is longer than LLIN lifespan which can account for a variation in reported LLIN use between the quintiles and between rural and urban areas. In addition, publicly funded mechanisms intended for sustaining high level LLIN coverage for vulnerable populations is delivered in formal sector sources whose access in rural areas and to poor populations in urban areas is narrow. Consequently, it is largely beneficial to relatively better off people concentrated in urban areas. 


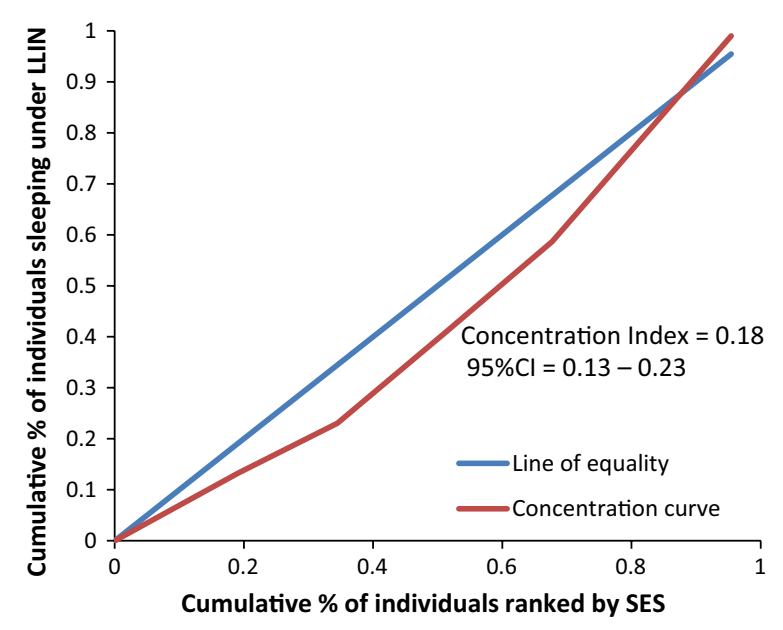

Fig. 11 Socio-economic inequality in LLIN use in Kibiti generated from the study

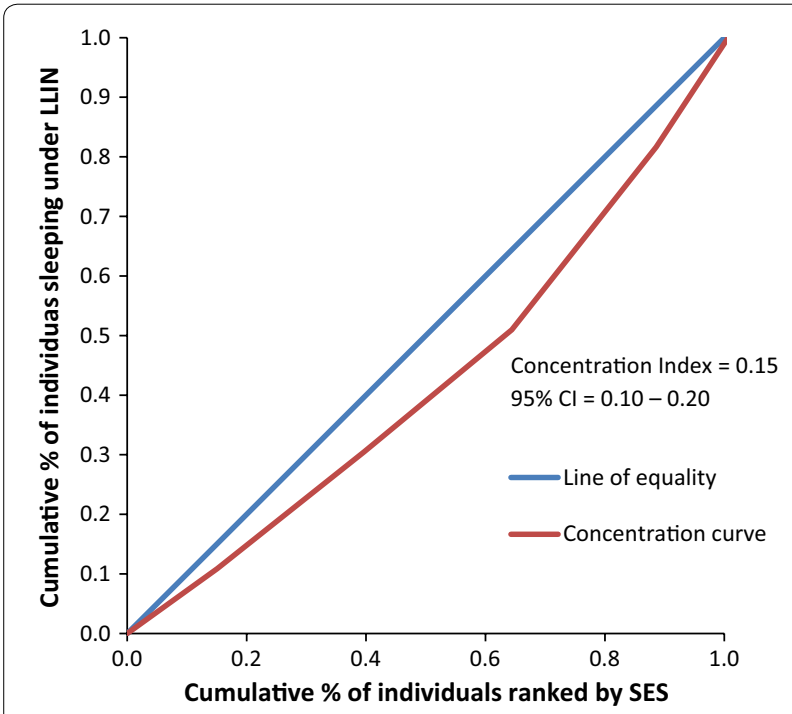

Fig. 12 Socio-economic inequality in LLIN use in Bungu generated from the study

\section{Conclusion}

The study has shown malaria parasite prevalence based on microscopic examination higher than the national average. It has shown substantial variations in parasitaemia between sub-district locations. This condition gives an impression that malaria control efforts in the study area have yielded varying impact on malaria burden. It appears that the burden is concentrated in areas and among groups with limited access to control interventions. It suggests that implementation of malaria control activities are favourable in easy-to-reach areas and to groups able to afford the costs involved in accessing

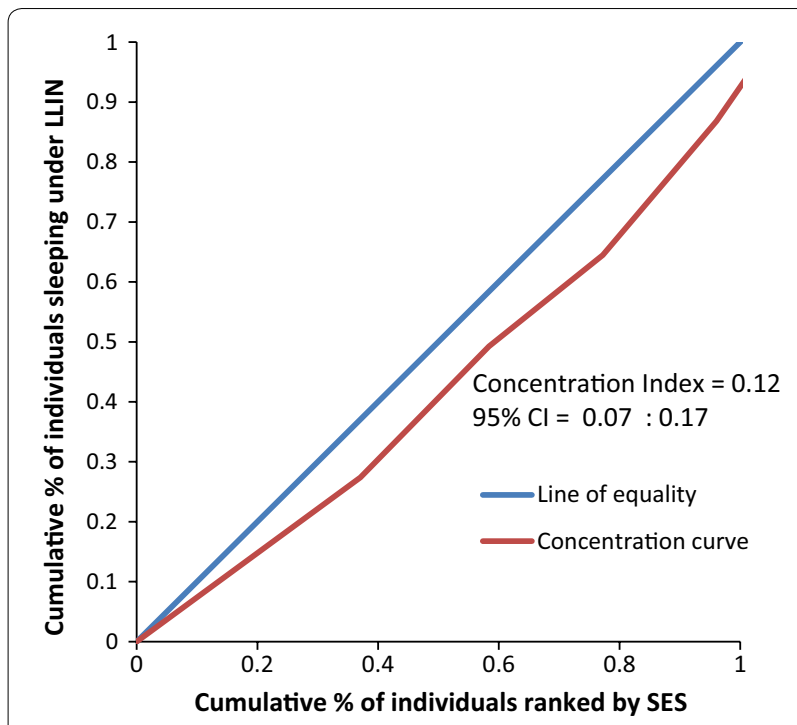

Fig. 13 Socio-economic inequality in LLIN use in Chumbi generated from the study

interventions targeted by publicly subsidized programmes. These are areas that are characterized by relatively good healthcare services that provide better access to such key malaria control interventions as LLINs and subsidized healthcare facilities. Population groups with economic strength can afford the high prices charged for malaria control interventions. The fact that LLIN use was highest in population centres, among under-fives, women, and the highest socio-economic quintile provides reasonable evidence to this conclusion.

It is evident that inaction is not an option for national malaria control programmes striving for progress in malaria control and malaria elimination. For success in malaria control in some areas and in some population groups, while leaving out peripheral areas and other marginal groups, will undo gains made and take the country back to where it was over a decade ago. It is important to identify and implement strategies that have worked elsewhere. Programmes that have worked to increase LLIN coverage for under-fives and pregnant women can be expanded to include school-aged children, adult males and non-pregnant females. The study has overall provided a broad picture of populations based parasite burden in Tanzania at sub-national level. However, its strength may have been in some ways been affected by the limitation of cross-sectional surveys. Data were collected in December and January, which are the peak season for malaria transmission in the area, but can hardly represent all of the remaining months of the year. Members of some sampled households could not be interviewed because they were not present at their 
homesteads during the time of the study. Their absence could have biased the results in some ways. Sample selection was based on households and therefore some population groups might have disproportionate representation and this would also have swayed the results.

\section{Author's contributions}

RAK contributed to the design of the study, supervised the field surveys, analysed and interpreted the data, and wrote the manuscript in consultation with the other authors. YPM, EK, TG and MGM participated in the design of the study, execution of the field surveys, interpretation of the data and editing of the manuscript. PCC, DW, NX, XZ and SA oversaw all aspects of the study, including design and execution of the field work, analysis and interpretation of the data and drafting of the manuscript. All authors read and approved the final manuscript.

\section{Author details}

1 Ifakara Health Institute, Kiko Avenue, Mikocheni, P.O. Box 78373, Dar es Salaam, United Republic of Tanzania. ${ }^{2}$ Swiss Tropical and Public Health Institute (Swiss TPH), Socinstrasse 57, P.O. Box, 4002 Basel, Switzerland. ${ }^{3}$ University of Basel, Petersplatz 1, 4003 Basel, Switzerland. ${ }^{4}$ National Institute of Parasitic Diseases, Chinese Center for Disease Control and Prevention, 207 Rui Jin Er Road, Shanghai 200025, People's Republic of China.

\section{Acknowledgements}

This study was based on China-Tanzania Joint Malaria Control Pilot Project implemented in Rufiji District, Coast Region, Tanzania. The authors wish to thank the many investigators, data collectors and field supervisors who contributed to the collection and analyses of these data including Dr. Weidong Li, Zhengbin Zhou, Jun Feng, He Yan, Kangming Li, Xiangli Kong, Yongbin Wang, Benguang Zhang, Xiaotao Zhao, Xucan Zeng, Xiaohong Wu, Irene Masanja, Tumaini Kilimba and Iddi Mkilalu. We would also acknowledge very important contribution of Dr. Honorati Masanja. We are especially grateful for the contributions of the Ministry of Health, Community Development, Gender, Elderly and Children (Tanzania), including the National Malaria Control Programme, National Institute for Medical Research (NIMR) and the Council Health Management Teams of Rufiji District together with China's Centers for Disease Control. Finally, we wish to acknowledge the community members who participated in or otherwise contributed to these studies.

\section{Competing interests}

The authors declare that they have no competing interests.

\section{Availability of data and materials}

The datasets used and/or analyzed during the current study are available from the corresponding author on reasonable request.

\section{Consent for publication}

Not applicable.

\section{Ethics approval and consent to participate}

Ethics approval for the study was granted by the institutional review boards of Ifakara Health Institute (IHI) (IHI/IRB/No: 18-2015, and by the National Tanzanian Medical Research Co-ordinating Committee of the National Institute for Medical Research (NIMR) (certificate No. NIMR/HQ/R.8a/Nol.IX/2015). Study participants were individually asked for written informed consent. For children less than 16 years old, the consent was obtained from the parent or guardian.

\section{Funding}

This study was Supported by China-UK Global Health Support Programme (GHSP-CS-OP4-D02) funded by UK DFID.

\section{Publisher's Note}

Springer Nature remains neutral with regard to jurisdictional claims in published maps and institutional affiliations.

\section{References}

1. Tanzania's Ministry of Health, Gender, Elderly and Children. Tanzania Demographic and Health Survey and Malaria Indicator Survey (TDHSMIS) 2015-2016; 2016. https://www.dhsprogram.com. Accessed 10 Apr 2018.

2. Lwetoijera DW, Harris C, Kiware SS, Dongus S, Devine GJ, McCall PJ, et al. Increasing role of Anopheles funestus and Anopheles arabiensis in malaria transmission in the Kilombero Valley, Tanzania. Malar J. 2014;13:331.

3. Killeen GF. Characterizing, controlling and eliminating residual malaria transmission. Malar J. 2014;13:330

4. Ministry of Health and Social Welfare. National Malaria Strategic Plan 2014-2020. Dar es Salaam: Ministry of Health and Social Welfare; 2014.

5. Kakwani NC, Wagstaff A, van Doorslaer E. Socio-economic inequalities in health: Measurement, computation and statistical inference. J Econometrics. 1997;77:87-104.

6. World Health Organization. World malaria report 2016. Geneva: World Health Organization; 2016.

7. Snow RW, Okiro EA, Gething PW, Atun R, Hay SI. Equity and adequacy of international donor assistance for global malaria control: an analysis of populations at risk and external funding commitments. Lancet. 2010;376:1409-16.

8. Galactionova K, Smith TA, de Savigny D, Penny MA. State of inequality in malaria intervention coverage in sub-Saharan African countries. BMC Med. 2017:15:185.

9. Gonçalves BP, Huang C-Y, Morrison R, Holte S, Kabyemela E, Prevots DR, et al. Parasite burden and severity of malaria in Tanzanian children. N Engl J Med. 2014:370:1799-808.

10. Ishengoma D, Segeja MD, Alifrangis M, Lemnge MM, Bygbjerg lb C. Declining burden of malaria over two decades in a rural community of Muheza district, north-eastern Tanzania. Malar J. 2013;12:388.

11. Taylor C, Florey L, Yé Y. Increasing equity of insecticide-treated net ownership in sub-Saharan Africa from 2003 to 2014. Bull World Health Organ. 2017;95:322-32.

12. Victora CG, Barros AJ, Axelson H, Bhutta ZA, Chopra M, França GV, et al. How changes in coverage affect equity in maternal and child health interventions in 35 Countdown to 2015 countries: an analysis of national surveys. Lancet. 2012;380:1149-56.

13. Teklehaimanot A, Mejia P. Malaria and poverty. Ann N Y Acad Sci. 2008;1136:32-7.

14. Khatib R, Skarbinski J, Njau JD, Goodman CA, Elling BF, Kahigwa E, et al. Routine delivery of artemisinin-based combination treatment at fixed health facilities reduces malaria prevalence in Tanzania: an observational study. Malar J. 2012;11:140.

15. Wang SJ, Lengeler C, Mtasiwa D, Mshana T, Manane L, Maro G, et al. Rapid Urban Malaria Appraisal (RUMA) II: epidemiology of urban malaria in Dar es Salaam (Tanzania). Malar J. 2006;5:28.

16. Masanja H. Malaria epidemiology in Tanzania; 2011. http://www.tzdpg.or. tz. Accessed 20 Apr 2018.

17. Bonner K, Mwita A, McElroy PD, Omari S, Mzava A, Lengeler C, et al. Design, implementation and evaluation of a national campaign to distribute nine million free LLINs to children under five years of age in Tanzania. Malar J. 2011;10:73.

18. Paaijmans KP, Blanford S, Bell AS, Blanford JI, Read AF, Thomas MB. Influence of climate on malaria transmission depends on daily temperature variation. Proc Natl Acad Sci USA. 2010;107:15135-9.

19. President's malaria initiative: malaria operational plan (MOP), Tanzania; 2012. https://www.pmi.gov. Accessed 13 Apr 2018.

20. Hawley WA, Phillips-Howard PA, ter Kuile FO, Terlouw DJ, Vulule JM, Ombok M, et al. Community-wide effects of permethrin-treated bednets on child mortality and malaria morbidity in western Kenya. Am J Trop Med Hyg. 2003;68(Supppl 4):121-7.

21. Killeen GF, Smith TA, Ferguson HM, Abdulla S, Mshinda H, Lengeler C, et al. Preventing childhood malaria in Africa by protecting adults from mosquitoes with insecticide-treated nets. PLoS Med. 2007;4:e229.

22. Khatib RA, Killeen GF, Abdulla SM, Kahigwa E, McElroy PD, Gerrets RP, et al, Markets, voucher subsidies and free nets combine to achieve high bed net coverage in rural Tanzania. Malar J. 2008;7:98.

23. Killeen GF, Govella NJ, Lwetoijera DW, Okumu FO. Most outdoor malaria transmission by behaviourally-resistant Anopheles arabiensis is mediated by mosquitoes that have previously been inside houses. Malar J. 2016;15:225. 
24. Eisele TP, Larsen DA, Walker N, Cibulskis RE, Yukich JO, Zikusooka CM, et al. Estimates of child deaths prevented from malaria prevention scale-up in Africa 2001-2010. Malar J. 2012;11:93.

25. Curtis C, Maxwell C, Lemnge M, Kilama W, Steketee RW, Hawley WA, et al. Scaling-up coverage with insecticide-treated nets against malaria in Africa: who should pay? Lancet Infect Dis. 2003;3:304-7.

26. Bhattarai A, Ali AS, Kachur SP, Mårtensson A, Abbas AK, Khatib R, et al. Impact of artemisinin-based combination therapy and insecticidetreated nets on malaria burden in Zanzibar. PLoS Med. 2007:4:e309.

27. Lynn M, Bossak B. Analysis of a secular trend in malaria incidence: Venezuela, 1959-2015. J Glob Epidemiol Environ Health. 2017;2017:54-9.

28. Aregawi M, Lynch M, Bekele W, Kebede H, Jima D, Taffese HS, et al. Time series analysis of trends in malaria cases and deaths at hospitals and the effect of antimalarial interventions, 2001-2011, Ethiopia. PLoS ONE. 2014;9:e106359.

29. National Malaria Control Programme (Tanzania), Ifakara Health Institute (Tanzania), KEMRI-Wellcome Trust (Kenya). An epidemiological profile of malaria and its control in Mainland Tanzania. Report funded by Roll Back Malaria and Department for International Development-UK; 2013.

30. Emami SN, Ranford-Cartwright LC, Ferguson HM. The transmission potential of malaria-infected mosquitoes (An. gambiae-Keele, An. arabiensisIfakara) is altered by the vertebrate blood type they consume during parasite development. Sci Rep. 2017;7:40520.

31. Bayoh MN, Mathias DK, Odiere MR, Mutuku FM, Kamau L, Gimnig JE, et al. Anopheles gambiae: historical population decline associated with regional distribution of insecticide-treated bed nets in western Nyanza Province, Kenya. Malar J. 2010;9:62.
32. Killeen GF, Smith TA. Exploring the contributions of bed nets, cattle, insecticides and excitorepellency to malaria control: a deterministic model of mosquito host-seeking behaviour and mortality. Trans R Soc Trop Med Hyg. 2007;101:867-80.

33. Killeen GF, Smith TA. Exploring the contributions of bednets, cattle, insecticides and excitorepellency to malaria control: a deterministic model of mosquito host-seeking behaviour and mortality. Trans R Soc Trop Med Hyg. 2007;101(9-4):867-88.

34. Okumu FO, Moore SJ. Combining indoor residual spraying and insecticide-treated nets for malaria control in Africa: a review of possible outcomes and an outline of suggestions for the future. Malar J. 2011;10:208.

35. Okumu FO, Kiware SS, Moore SJ, Killeen GF. Mathematical evaluation of community level impact of combining bed nets and indoor residual spraying upon malaria transmission in areas where the main vectors are Anopheles arabiensis mosquitoes. Parasit Vectors. 2013;6:17.

36. Alonso PL, Bell D, Hanson K, Mendis K, Newman RD, de Savigny D, et al. A research agenda for malaria eradication: health systems and operational research. PLoS Med. 2011:8:e1000397.

37. Khatib RA, Selemani M, Abdalla G, Masanja I, Amuri M, Njozi M, et al. Access to artemisinin-based anti-malaria treatment and its related factors in rural Tanzania Dar es Salaam 2012. Malar J. 2013;12:155

38. Gallup JL, Sachs JD. The economic burden of malaria. Am J Trop Med Hyg. 2001;64:85-96.

39. Russell S. The economic burden of illness for households in developing countries: a review of studies focusing on malaria, tuberculosis, and human immunodeficiency virus/acquired immunodeficiency syndrome. Am J Trop Med Hyg. 2004;71:147-55.
Ready to submit your research? Choose BMC and benefit from:

- fast, convenient online submission

- thorough peer review by experienced researchers in your field

- rapid publication on acceptance

- support for research data, including large and complex data types

- gold Open Access which fosters wider collaboration and increased citations

- maximum visibility for your research: over 100M website views per year

At BMC, research is always in progress.

Learn more biomedcentral.com/submissions 